\title{
DELIRIUM APRES CHIRURGIE AVEC CIRCULATION EXTRA-CORPORELLE
}

\author{
Pierre Coupal, Pierre Morin et Bernard Paiement
}

\begin{abstract}
RÉSUMÉ
La littérature récente rapporte encore une incidence élevée de delirium après chirurgie avec circulation extra-corporelle. Ce syndrome que l'on est plus susceptible de rencontrer chez les malades de plus de 50 ans, avec une histoire d'atteinte cérébrale antérieure ( $\mathrm{ACV}$, histoire de delirium, d'alcoolisme ou de toxicomanie) et que l'on observe plus fréquemment après une chirurgie valvulaire que chez les coronariens, est surtout caractérisé par des modifications de l'attention et de la concentration, avec ou sans perceptions inadéquates, langage incohérent, insomnie, agitation ou apathie. Il s'accompagne également de désorientation et de troubles de la mémoire. Il apparaît précocement après la chirurgie, connaît une évolution fluctuante et le plus souvent bénigne. On est plus susceptible de le rencontrer chez les malades présentant des complications post-opératoires avec atteinte importante de l'état général.

Au cours d'une étude rétrospective portant sur les quatre dernières années à l'Institut de Cardiologie de Montréal, on a relevé 84 cas sur 2811 patients soumis à une chirurgie extracorporelle (2.98 pour cent). L'âge moyen des malades ayant présenté un delirium était de 57.5 ans (versus 50.4 ans) et l'on a retrouvé des complications post-opératoires importantes chez 37 ( 44 pour cent) de ces patients. L'incidence était moins élevée chez les malades soumis à une chirurgie coronarienne ( 1.45 pour cent) que chez ceux ayant subi un remplacement valvulaire (4.6 pour cent). Différents facteurs pouvant expliquer cette basse incidence sont discutés.
\end{abstract}

Mots Clefs: Complications, delerium; Circulation extra-corporelle.

L'APPELLATION PSYCHOSE post-opératoire est couramment utilisée en clinique. Les psychiatres préfêrent parler, dans ces cas, de delirium postopératoire, un terme qui correspond mieux à la réalité clinique. Alors que le malade en psychose a perdu le contact avec la réalité, le patient en delirium présente une atteinte des fonctions mentales supérieures, notamment de la capacité d'attention et de concentration, il n'a pas nécessairement perdu le contact avec la réalité.

Ce sont Blackly et Starr ${ }^{1}$ qui, en 1964, ont été les premiers à utiliser le terme de delirium post-cardiotomie. Par la suite, de nombreux auteurs ont proposé leurs critères de définition du syndrome et ont décrit diverses formes cliniques, ${ }^{2.3}$ ce qui a conduit à une grande confusion. Cette confusion persiste encore malgré les efforts récents pour en arriver à un consensus dans la définition du syndrome et la terminologie.

Ce travail a pour but: $1^{0}$ de présenter une brève

Pierre Coupal, M.D., F.R.C.P. (C), Psychosomaticien consultant, Institut de Cardiologie de Montréal; Pierre Morin, M.D., F.R.C.P. (C), Professeur Adjoint de Clinique, Département de Psychiatrie, Université de Montreal; Bernard Paiement, M.D., F.R.C.P. (C), Professeur Agrégé de Clinique, Département d'Anesthésie-Réanimation, Université de Montréal et Institut de Cardiologie de Montréal, 5000 est, rue Bélanger, Montréal, Québec HIT 1C8. revue de cette entité clinique et de son traitement et $2^{\circ}$ de rapporter l'expérience de l'Institut de Cardiologie de Montréal dans ce domaine au cours des quatre dernières années.

\section{Description Clinioue}

Nous avons proposé ${ }^{4}$ de regrouper dans un même ensemble tous les cas de delirium survenant après une intervention chirurgicale avec emploi de la circulation extra-corporelle (CEC), avec ou sans cardiotomie, que le malade ait présenté ou non un intervalle lucide après la chirurgie. Les réactions émotionnelles de dépression ou d'anxiété plus ou moins importantes et plus ou moins normales, communément observées, n'ont pas été incluses dans notre définition du syndrome.

Nous posons le diagnostic de delirium ${ }^{4,5}$ après chirurgie avec $\mathrm{CEC}$ lorsque nous retrouvons chez un malade:

A. Une atteinte de l'attention et de la concentration qui sont le plus souvent diminuées, mais peuvent être accentuées dans les états de panique.

B. Deux des quatre critères suivants:

1. Perceptions inadéquates: illusions, hallucinations (visuelles le plus souvent: arai- 
gnées, serpents, petits hommes ...), idées interprétatives et incapacité de distinguer le rêve de la réalité.

2. Langage incohérent.

3. Perturbations du cycle éveil-sommeil avec insomnie ou assoupissement diurne.

4. Activité psycho-motrice augmentée ou diminuée (agitation, apathie).

C. De la désorientation, surtout dans le temps, mais parfois dans l'espace et, plus rarement, quant aux personnes. Le patient se trompe de mois, se pense chez lui ou dans un autre hôpital, ne reconnait plus certains membres de sa famille.

D. Des troubles de la mémoire, surtout des faits récents (le malade ne se rappelle pas d'avoir été opéré).

E. Une apparition rapide des symptômes qui s'installent en quelques jours ou quelques heures après la chirurgie.

F. Un syndrome à évolution fluctuante.

Certains malades ne présentent pas toutes ces caractéristiques. Ainsi, nous avons observé chez quelques malades la présence d'hallucinations visuelles avec une attention augmentée et un état de méfiance de type paranoïde, sans désorientation ni atteinte de la mémoire des faits récents. Nous avons quand même regroupé ces patients avec les cas plus classiques de delirium, les critères diagnostiques ne faisant pas l'objet d'un consensus dans la littérature. ${ }^{3-9}$

Classiquement, l'évolution est la suivante: quelques jours après l'opération, le malade présente une attention moins précise, semble anxieux, son comportement est différent et ceux qui le connaissent bien trouvent qu'il a "l'air drôle", il oublie les événements récents, est désorienté dans le temps, se trompe de jour de la semaine ou même de mois, se croit chez lui ou dans un autre hôpital et se méprend sur l'identité de certaines personnes; il tient des propos délirants, se sent persécuté, croit qu'une catastrophe le menace et présente souvent, surtout la nuit, des illusions ou des hallucinations qui l'inquiètent. Selon le contenu de ses hallucinations et de ses convictions délirantes, il peut devenir très agité, arracher ses cathéters et ses solutés, frapper les infirmières, etc ...

Il arrive qu'en raison d'un comportement très apathique chez certains patients, le diagnostic passe inaperçu, à moins qu'un examen attentif des fonctions mentales ne révèle ce que le malade ne confie pas spontanément (peur de passer pour fou s'il révèle ses hallucinations).

Habituellement, l'état du malade s'améliore rapidement, en quelques jours, et ce, avec ou sans traitement, bien que ce dernier semble hâter la récupération et prévenir certaines complications.

\section{INCIDENCE}

L'incidence rapportée de delirium après chirurgie avec CEC est élevée: elle varie, selon les publications, de 13 à 67 pour cent ${ }^{3.8 .10}$ des cas. Elle serait plus élevée dans les cas de chirurgie valvulaire qu'en chirurgie coronarienne ${ }^{10.11}$.

\section{ErIOLOGIF}

L'étiologie exacte est inconnue. De nombreux facteurs semblent cependant prédisposer les malades au syndrome ou le déclencher.

\section{Facteurs pré-opératoires}

1. Age: Les patients de plus de 50 ans sont plus susceptibles de présenter des delirium. ${ }^{3.8}$

2. On en retrouverait davantage chez les malades avec une histoire d'atteinte antérieure de la fonction cérébrale, soit par un accident cérébrovasculaire, par l'alcoolisme ou une toxicomanie ou par un épisode de delirium, quelle qu'en soit l'origine..$^{5-7,12,13}$

Ces deux facteurs sont les plus importants pour dépister avant l'intervention les malades nécessitant une attention spéciale. D'autres facteurs ont également été mentionnés mais sont moins faciles à démontrer, à savoir:

3. Un état d'anxiété, de peur ou de dépression marquée avant l'intervention, surtout lorsque les malades ne l'expriment pas ou prennent panique. ${ }^{3,8,9,12,14}$ Une acceptation stoïque peut masquer une négation dangereuse..$^{7,9,14}$

4. Une mauvaise préparation à la chirurgie ou une absence d'informations pré-opératoires; ${ }^{8}$ une intervention effectuée en urgence sans que le patient ait eu le temps de se faire à cette idée.

5. Une intervention remise à plusieurs reprises: le malade se plaint que l'on "joue avec ses nerfs".

\section{Facteurs per-opératoires}

De nombreux facteurs per-opératoires ont également été mis en cause, comme la complexité et la durée de l'intervention, la durée de la $\mathrm{CEC},{ }^{3,6,10}$ l'hypotension per-opératoire, ${ }^{15.16} \mathrm{des}$ micro-embolies ou de l'hypoperfusion en cours de $\mathrm{CEC},{ }^{15.16}$ des phénomènes emboliques (caillots, calcium, air) avec dommages cérébraux, des 
troubles du rythme graves, l'arrêt cardiaque, les hémorragies importantes et les difficultés du réchauffement en phase post-opératoire immédiate..$^{3,8,9}$

\section{Facteurs post-opératoires}

La présence de complications post-opératoires graves avec atteinte importante de l'état général constitue le principal facteur de déclenchement d'un delirium. Ainsi, un syndrome de bas débit post-opératoire avec nécessité d'utiliser la contrepulsation par ballon intra-aortique représente une situation susceptible de conduire à un delirium.

D'autres facteurs secondaires ont également été proposés:

1. Des difficultés dans les relations interpersonnelles des médecins, infirmières et autres membres de l'équipe soignante, conduisant à un climat de tension et de mésentente dans l'entourage du patient.

2. La déprivation sensorielle dans les unités dépourvues de fenêtres, ${ }^{17.18}$ le malade ne pouvant distinguer le jour de la nuit et recevant constamment les stimuli monotones de l'appareillage.

3. Un sommeil troublé par les interventions fréquentes du personnel ${ }^{19}$ (prise des signes vitaux et soins) soit chez lui ou chez les malades voisins ou, par un aménagement des locaux ne procurant pas un minimum d'intimité aux malades. ${ }^{14}$ Cependant, même lorsque les conditions sont optimales, on retrouve plus de perturbations du sommeil chez les patients ayant subi une intervention cardiaque que dans les cas de chirurgie abdominale.

\section{Pronostic}

Une mortalité et une morbidité plus élevées ont été rapportées chez les malades ayant présenté un delirium. ${ }^{13}$ Les études prospectives en ce domaine sont rares et, comme nous l'avons déjà mentionné, on sait que l'on retrouve une incidence plus élevée de delirium chez les malades ayant présenté des complications graves après l'intervention ...

Le pronostic est en général excellent: le malade récupère rapidement et complètement, sauf lorsqu'il y a dommage cérébral irréversible.

\section{Prévention}

Une bonne préparation à la chirurgie cardiaque implique:

1. Le dépistage des patients à haut risque de présenter un delirium.

2. L'établissement d'une relation inter-person- nelle satisfaisante entre le malade et l'équipe qui le traite. Un rapport personnalisé, chaleureux et supportif procure au malade le sentiment de sa propre valeur. Une attitude stéréotypée, centrée sur la dimension mécanique et technique de l'intervention ne peut jouer un rôle préventif.

3. Un personnel conscient des facteurs de risque du delirium, peut mieux préparer le malade à la chirurgie en établissant un climat de confiance et d'échange et, après la chirurgie, sait mieux prévenir, dépister et traiter la complication lorsqu'elle survient.

4. Le recours à un consultant spécialisé ne devrait être utilisé que chez les malades où le risque de delirium est élevé et, dans de telles situations, le spécialiste devrait bénéficier de suffisamment de temps pour préparer le malade. Plusieurs approches thérapeutiques ${ }^{3,6,8}$ sont disponibles.

\section{TRAITEMENT}

\section{Attitude}

Il est important de resituer un patient qui a

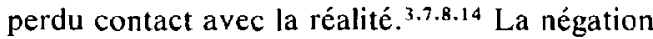
des propos délirants peut augmenter l'agitation ou favoriser le retrait du malade. Un éclairage discret de la chambre favorise le repos et prévient la survenue des illusions et des hallucinations. ${ }^{8,14,18}$ L'usage d'objets familiers (horloge, calendrier, photographies) contribue à resituer le patient dans le temps et l'espace.

Une surveillance plus étroite s'impose sans recours abusif aux contentions (qui peuvent favoriser l'agitation). Un effort doit être fait pour conserver le même personnel auprès du patient, ce qui permet de mieux suivre l'évolution du comportement et de le rassurer par la continuité d'une image familière.

Le recours à la famille est précieux et permet de combler les lacunes du système hospitalier.

Enfin, il est primordial de rassurer le malade sur son état dès qu'il est en mesure de comprendre. ${ }^{3,7,9,14}$

\section{Médication}

Outre la correction des déséquilibres biologiques pouvant contribuer au delirium, il est indiqué d'utiliser une médication psychotrope pour diminuer l'agitation, la panique, les hallucinations et la perte de contact avec la réalité. ${ }^{7-9,12.14 .20}$ On recommande un neuroleptique. Les tranquillisants mineurs, comme les benzodiazépines, sont d'une efficacité discutable.

L'halopéridol (Haldol) est le neuroleptique de choix. Ce dérivé de la butyrophénone présente moins d'effets anticholinergiques et cardio-vas- 
culaires, est moins sédatif, plus efficace et plus rapide pour faire cesser le délire et diminuer l'agitation que les phénothiazines (chlorpromazine). Son mode d'action étant lié à une inhibition des récepteurs dopaminergiques au niveau du $\mathrm{SNC}$, on ne recommande pas son usage lorsque l'on doit utiliser de l'épinéphrine.

Les patients en delirium répondent à des doses relativement faibles d'halopéridol. La posologie varie de 3 à $10 \mathrm{mg}$ die, administrés en deux, trois ou quatre doses fractionnées. On le prescrit à raison de 1 à $2 \mathrm{mg}$ t.i.d. ou q.i.d., le dosage étant augmenté ou diminué graduellement selon la réponse du malade. Par voie intra-musculaire, on réduit la dose de moitié. L'halopéridol devrait être diminué graduellement (en quelques jours) dès que l'état du malade le permet et, il devrait être discontinué avant la sortie de l'hôpital.

On propose de l'associer à un anti-parkinsonien, afin de contrôler les effets extra-pyramidaux observés fréquemment avec ce médicament. La procyclidine (Kemadrin) per os, à la dose de $2.5 \mathrm{mg}$, b.i.d. ou t.i.d. est efficace.

Une attention spéciale devrait être portée aux médicaments d'usage courant en phase postopératoire. ${ }^{20}$ Plusieurs d'entre eux (analgésiques, hypnotiques, anti-nauséeux, cimétidine (Tagamet), méthylphénidate (Ritalin)) peuvent précipiter ou aggraver un delirium, potentialiser ou réduire l'efficacité des neuroleptiques ou augmenter leurs effets secondaires.

\section{Delirium après Chirurgie Cardiaque avec CEC À l'INSTITUT de CARDiologie de Montréal}

Une étude rétrospective des cas de delirium après CEC survenus à l'Institut de Cardiologie de Montréal au cours des quatre dernières années a été effectuée. ${ }^{4}$ Les infirmières des unités postopératoires de cette institution sont bien informées de ce type de complications et rapportent systématiquement au médecin traitant tout état mental s'éloignant de la normale. Une consultation au psychosomaticien est alors demandée de façon systématique. Tous les cas reconnus, sauf un, ont donc été vus en consultation, et traités, par les psychosomaticiens au cours des quatre dernières années. Les tableaux I et II résument notre expérience.

\section{RÉSULTATS}

Nous avons observé 84 cas de delirium sur 2811 patients ayant subi une chirurgie avec CEC, soit une incidence de 2.98 pour cent. L'âge moyen des patients ayant présenté un delirium était de 57.5 ans, alors que celui de l'ensemble de nos cas de CEC était de 50.4 ans. Des complications post-opératoires graves (bas débits nécessitant une contre-pulsation intra-aortique, insuffisance respiratoire, infections) ont été observées chez 37 (44 pour cent) de ces 84 patients.

Nos résultats de la dernière année (Tableau II) montrent une incidence moins éle vée de delirium chez les malades soumis à une chirurgie coronarienne ( 7 cas/483-1.45 pour cent) que chez les malades ayant subi un remplacement valvulaire (11 cas/239-4.6 pour cent) ce qui correspond aux données de la littérature.

\section{Discussion}

Notre incidence de 2.8 pour cent de delirium après chirurgie avec circulation extra-corporelle est inférieure aux chiffres cités dans la littérature récente, ${ }^{3,9-11}$ Plusieurs facteurs méritent d'être soulignés pour expliquer ce résultat:

1. Notre étude a été effectuée de façon rétrospective, ce qui est moins valable qu'une étude prospective.

2. Nous avons exclu tous les syndromes affectifs réactionnels sans signes d'atteinte cérébrale organique. Par ailleurs, nos critères diagnostiques ont été élargis pour inclure les malades ayant présenté des hallucinations visuelles ou une attention augmentée sans atteinte des autres fonctions mentales supérieures.

3. La probabilité d'un inventaire incomplet existe bien que le personnel médical et paramédical soit tenu bien au courant de cette entité et sache la reconnaitre précocement.

4. La préparation des malades à l'intervention fait l'objet d'une attention spéciale. Un effort collectif est fait pour établir un climat de confiance entre le malade et ses médecins et ses infirmières (enseignement pré-opératoire planifié) et les physiothérapeutes au besoin.

5. Nos résultats reflètent la pratique des dernières années, alors que les techniques chirurgicales et anesthésiques se sont beaucoup améliorées par des meilleures techniques de protection myocardique en cours de $\mathrm{CEC}$, cardioplégie, ${ }^{21,22}$ réalisation de l'importance du maintien d'une pression-de perfusion adéquate, ${ }^{16}$ meilleur monitoring péri-opératoire (généralisation de l'emploi des cathéters de Swan-Ganz), usage per et postopératoire des bêta-bloquants et des vasodilatateurs. ${ }^{23}$ L'amélioration des techniques permet maintenant à de nombreux centres de rapporter des mortalités globales de 3 à 5 pour cent pour la chirurgie cardiaque générale et de 1 à 2 pour cent pour la chirurgie coronarienne. ${ }^{24-27}$ 
TABLEAU I

Delirium Post-Chirurgie Cardiaque avec CEC à l'Institut de Cardiologie de Montréal entre Avril 1976 et Avril 1980

\begin{tabular}{ccccc}
\hline \hline & $\begin{array}{c}\text { Nombre de cas } \\
\text { de delirium/ } \\
\text { Nombre de cas } \\
\text { opérés (CEC) }\end{array}$ & Incidence & $\begin{array}{c}\text { Nombre de cas } \\
\text { de delirium } \\
\text { Age moyen des } \\
\text { cas de delirium/ } \\
\text { Age moyen des } \\
\text { opérés (CEC) } \\
\text { post-op. graves } \\
\text { (infection, ballon, } \\
\text { insuff. respiratoire) }\end{array}$ \\
\hline $1976-1977$ & $16 / 539$ & $2.97 \%$ & $53.5 / 48.8$ & $8 / 16$ \\
$1977-1978$ & $18 / 720$ & $2.5 \%$ & $58.11 / 51.1$ & $10 / 18$ \\
$1978-1979$ & $31 / 759$ & $4.08 \%$ & $58.51 / 50.2$ & $12 / 31$ \\
$1979-1980$ & $19 / 793$ & $2.39 \%$ & $58.52 / 51.9$ & $7 / 19$ \\
Total & $* 84 / 2811$ & $2.98 \%$ & $57.5 / 50.4$ & $37 / 84$ \\
\hline
\end{tabular}

*Dont 83 vus en consultation.

TABLEAU II

RÉpartition de L'INCIDENCE SUIVANT LE TYPE d'INTERVENTION EN 1979-1980

\begin{tabular}{lccc}
\hline \hline \multicolumn{1}{c}{ Type d'intervention } & $\begin{array}{c}\text { Total des } \\
\text { opérés }\end{array}$ & $\begin{array}{c}\text { Cas de } \\
\text { delirium }\end{array}$ & Incidence \\
\hline Pontage aorto-coronarien & 483 & 7 & $1.45 \%$ \\
Remplacement valvulaire & 239 & 11 & $4.6 \%$ \\
Résection d'anévrysme & 42 & 1 & $2.38 \%$ \\
Congénitaux & 26 & - & - \\
Divers & 3 & - & - \\
$\quad$ Total & 793 & 19 & $2.39 \%$ \\
\hline
\end{tabular}

N.B. Les patients ayant subi et remplacement valvulaire et pontage ont été regroupés dans la section des remplacements valvulaires. Les patients ayant subi pontage et résection d'anévrysme ont été regroupés dans la section des résections d'anévrysme.

La morbidité opératoire a également diminué comme en témoigne le fait que dans notre milieu comme dans de nombreux centres, la majorité des malades ne passe que 18 à 36 heures aux soins intensifs. De même, de nombreuses équipes ne jugent plus nécessaire de maintenir une politique de ventilation mécanique chez tous les opérés au cours de la première nuit, préférant juger chaque cas au mérite. Le fait qu une proportion importante de malades connaisse des suites postopératoires sans complications graves, explique que l'incidence de delirium soit réduite.

6. L'emploi systématique de diazepam au moment de l'induction et durant la ventilation mécanique post-opératoire explique que plus de la moitié des patients n'ont aucun souvenir de leur arrivée et de leur séjour au bloc opératoire et de fait, ne se rappellent de rien du tout jusqu'au lendemain de l'intervention. ${ }^{28}$

7. Finalement, l'évaluation précoce des facteurs de risque permet une intervention avant l'éclosion du delirium.

\section{Conclusion}

La nécessité de vérifier de façon prospective l'incidence des cas de delirium à l'Institut de Cardiologie de Montréal nous semble impérative. Si l'incidence demeure inférieure par un facteur de deux à trois à l'incidence rapportée dans la littérature, il sera alors possible de départager l'importance des différents facteurs mentionnés dans la discussion. Il apparaît également nécessaire de surveiller l'évolution de ce concept dans la littérature. Nous souhaitons une standardisation des critères diagnostiques pour permettre une meilleure comparaison des résultats entre les centres.

\section{REFERENCES}

1. Blackly, P.H. \& Starr, A. Post-cardiotomy delirium. Am. J. Psychiatry 121: 371-375 (1964)

2. Tufo, H.M., Ostfeld, A.M. \& Shekelle, R. Central nervous system dysfunction following openheart surgery. J. Am. Med. Assoc. 212: 1333-1340 (1970). 
3. Speidel, H., Dahme, B. \& Flemming, B. Openheart surgery unit. Adv. Psychosom. Med. 10: $30-56(1980)$.

4. Morin, P. \& Coupal, P. Delirium post-chirurgie cardiaque avec circulation extra-corporelle: aspects cliniques et observations dans un centre spécialisé. Can. J. Psychiatry. A paraître. Accepté pour publication 1981.

5. American Psychiatric Association. Diagnostic and statistical manual of mental disorders (Third Edition), pp. 104-107 (1980).

6. Kimball, C.P. The experience of open-heart surgery. IIl. Toward a definition and understanding of post-cardiotomy delirium. Arch. Gen. Psychiatry 27: 57-63 (1972).

7. Heller, S.S. \& Kornfeld, D.S. Delirium and related problems, In: American Handbook of Psychiatry, vol. 4, pp. 43-66, Arieti, S. (Ed-in-Chief) \& Reiser, M.F. (Ed.), Basic Books, N.Y. (1975).

8. VAsQuez, F. \& ChITwood, W.R. Postcardiotomy delirium: an overview. Int. L.J. Psychiatric, In: Medicine 6: 373-383 (1975).

9. Dubin, W.R., Field, H.L. \& GastFriend, D.R. Postcardiotomy delirium: A critical review. J. Thorac. Cardiovasc. Surg. 77: 586-594 (1979).

10. Kornfeld, D.S., Heller, S.S. \& Frank, K.A. Delirium after coronary artery bypass surgery. J. Thorac. Cardiovasc. Surg. 76: 93-96 (1978).

11. Heller, S.S., Kornfeld, D.S., \& Frank, K.A Postcardiotomy delirium and cardiac output. Am. J. Psychiatry 136: 337-339 (1979).

12. Nadelson, T. The psychiatrist in the surgical intensive care unit. I. Post-operative delirium. Arch. Surg. 3: 113-119 (1976)

13. WILlNER, A.E. \& RABINER, C.J. Psychopathology and cognitive dysfunction: five years after openheart surgery. Comprehensive Psychiatry 20:409418 (1979).

14. Thompson, T.L. \& Feinberc, L.E. Evaluation of postoperative changes in mental status. Postgrad. Med. 67: 277-287 (1980).

15. Salerno, T.A., Lence, D.P. \& White, D.N. Monitoring of electroencephalogram during open heart surgery, A prospective analysis of 118 cases. J. Thorac. Cardiovasc. Surg. 76: 97-100 (1978).
16. Stockard, J.J., Bickford, R.G. \& Schauble, J.F. Pressure-dependent cerebral ischemia during cardiopulmonary bypass. Neurology 23: 521-529 (1973).

17. Engel, G.L. \& Romano, J. Delirium: a syndrome of cerebral insufficiency. J. Chronic. Dis. 9: 260277 (1959).

18. WILson, L.M. \& Dorado, E. Intensive care delirium. The effect of outside deprivation in a windowless unit. Arch. Intern. Med. 130: 225-226 (1972).

19. Johns, M.W., LARge, A.A. \& Masterton, J.P. Sleep and delirium after open-heart surgery. $\mathrm{Br}$. J. Surg. 61: 377-381 (1974)

20. MCNichol, R.W. \& Hoshino, A.Y. Therapy for deliria. Current Psychiatric Therapies, pp. 181-193 (1975).

21. Kirklin, J.W., Conti, V.R. \& Blackstone, E.H. Prevention of myocardial damage during cardiac operations. New Engl. J. Med. 301: 135141 (1979).

22. BuCKBERG, G.D. A proposed "solution" to the cardioplegic controversy. J. Thorac. Cardiovasc. Surg. 77: 803, 1977.

23. Paiement, B., Boulanger, M., Tailleffer, J. \& MaILle, J.G. Anesthésie et cardiophathie: évolution des concepts et perspectives. Union Médicale I08: 1158-1166 (1979).

24. BoncheK, L.I. Indications for surgery of the mitral valve. Am. J. Cardiol. 46: 155-158 (1980).

25. Fowler, N.O. \& VAN der Bel-KaHN, J.M. Operations on the mitral valve: time for weighing the issues. Am. J. Cardiol. 46: 159-161 (1980).

26. Kouchoukos, N.T., Oberman, A., Kirklin, J.W., Russell, R.O., Karp, R.B., Pacifico, A.D. \& ZoRN, G.L. Coronary bypass surgery: analysis of factors affecting hospital mortality. Circulation (suppl I): 62: 84-89 (1980).

27. Jamieson, W.R.E., Thompson, D.M. \& Munro, A.I.: Cardiac valve replacement in elderly patients. C.M.A.J. 123: 628-632 (1980).

28. Paiement, B., Boulanger, M., Jones, C.W. \& RoY, M. Intubation and other experiences in cardiac surgery: the consumer's views Canad. Anaesth. Soc. J. 26: 173-180 (1979).

\section{ABSTRACT}

The recent literature still reports a high incidence of delirium in patients operated with extracorporeal circulation. This syndrome is found more often in patients over 50 years and in patients with a previous history of delirium, alcoholism, drug addiction or cerebral damage and is more frequent after valvular than after coronary surgery. It is also often observed in patients who present major post-operative complications.

Attention and concentration capacity are characteristically modified (diminished most of the time) with or without faulty perception (hallucinations), sleep alterations, incoherent language, apathy or agitation. Disorientation and memory troubles are common. Delirium appears within a few hours or a few days of the surgery, its evolution is fluctuant and generally benign.

A retrospective study at the Montreal Heart Institute showed an incidence of 2.98 per cent (84 cases/2811 open heart surgery cases) over the last four years. The mean age of these patients was 57.5 years (versus 50.4 for patients without delirium). Major post-operative complications were found in 37 (44 per cent) of these 84 patients. The incidence was lower ( 1.45 per cent) in coronary than in valvular ( 4.6 per cent) surgery cases. Several explanations are presented to explain this low incidence.

Key Words: Complications, delerium; Extra-corporeal circulation. 